\title{
ORIGINAI
}

\section{EVALUACIÓN DE LA EFECTIVIDAD DE UN PROGRAMA DE DETECCIÓN DE INFECCIÓN ASINTOMÁTICA POR CHLAMYDIA TRACHOMATIS EN MUJERES}

\author{
Javier Jorge Mariñas, María Elena Rego, Miguel Rosales, María Isabel Castro y Encarnación \\ Bouzas.
}

Servicio de Medicina Preventiva y Salud Pública. Hospital Juan Canalejo. La Coruña.

\section{RESUMEN}

Fundamento: Chlamydia Trachomatis es actualmente el patógeno más prevalente de los causantes de Enfermedades de transmisión sexual en países desarrollados, alcanzando en alguros de ellos proporciones epidémicas.

Dichas infecciones pueden dar lugar a un considerable número de complicaciones, muchas de ellas de importantes consecuencias. Dado que además, un gran número de casos cursan de manera asintomática, existe un consenso generalizado en la conveniencia de realizar programas de cribaje.

El objetivo de este estudio es llevar a cabo la primera fase en la evaluación del programa de cribaje de infección asintomática por $\mathrm{C}$. trachomatis en las mujeres que acuden a un centro de planificación familiar.

Métodos: Estudio descriptivo para determinar la prevalencia de cada año del período estudiado, análisis de la tendencia temporal de la misma y valoración de la efectividad del programa en términos de reducción proporcional de prevalencia. Se incluyeron un total de 6.746 mujeres que acudieron al centro de planificación familiar para revisión ordinaria 0 consejo de anticoncepción entre enero de 1991 y diciembre de 1993

Resultados: El programa mostró una efectividad global del $80 \%$, reduciéndose la prevalencia de $5.1 \%$ en el año 90 a $1.0 \%$ en el año 93. Se observó un descenso significativo en todas las categorías analizadas, a excepción de laș mujeres que utilizaban preservativo y las tituladas superiores.

Conclusiones: El programa alcanza sus objetivos con una alta efectividad. No obstante, sería aconsejable realizar una valoración de su eficiencia incluyendo el análisis de los costes, ya que al disminuir la prevalencia el valor predictivo positivo de la prueba disminuye notablemente, lo que encarece la detección de un caso.

Palabras clave: Chlamydia trachomatis. Programa. Cribaje. Efectividad.

\section{Correspondencia:}

Javier Jorge Mariñas.

Servicio de Medicina Preventiva y Salud Pública.

Hospital Juan Canalejo.

As Xubias, s/n.

15006 La Coruña.

\section{ABSTRACT \\ Evaluation of the Effecdtiveness of a Detection Programme of Asymptomatic Infection by Chlamydia Trachomatis in Women}

Background: Chlamydia Trachomatis is at present the most prevalent pathogenic agent among the causes of sexually transmitted diseases in developed countries, and has reached epidemic proportions in some of them.

These infections can give rise to a considerable number of complications, many with very serious consequences. Given that, in addition, a great number of cases are asymptomatic, a general consensus exists about the convenience of carrying out screening programmes.

The aim of this study is to accomplish the first stage of the screening programme of asymptomatic infection by C. trachomatis in women who seek help at a family planning centre.

Methods: A descriptive study to determine the prevalence each year of the period studied, an analysis of its temporal tendency and the assessment of the programme effectiveness in terms of a proportional reduction in prevalence. A total number of 6.746 women were included, who attended the familiy planning centre for a regular checkup or contraceptive advice between January 1991 and December 1993.

Results: The programme showed a global effectiveness rate of $80 \%$, with a reduction in prevalence from $5,1 \%$ in 1990 to $1,0 \%$ in 1993. A significant fall was observed in all the categories analyzed, except for women using prophylactics and those with higher education.

Conclusions: The programme achieves its objectives very effectively. Nevertheless, it would be advisable to valuate its efficiency together with a cost analysis, since by reducing prevalence, the positive estimated value of the test considerably decreases, which raises the cost of a single case detection.

KEY WORDS: Chlamydia Trachomatis. Programme. Screening. Effectiveness. 


\section{INTRODUCCIÓN}

Chlamydia trachomatis (CT) es hoy en día el patógeno más importante y prevalente de los causantes de enfermedades de transmisión sexual en Europa, donde ha alcanzado ya proporciones cpidémicas, estimándosc que en algunos países un tercio de la población joven pueden tener una o más infecciones por dicho germen a lo largo de su vida, e igualmente en Estados Unidos donde es responsable cada año de cuatro millones de infecciones nuevas aproximadamente ${ }^{1-3}$.

En las mujeres, la mayoría de las infecciones por CT cursan de manera totalmente asintomática, $o$ a lo sumo con un cuadro anodino e inespecífico, que muchas veces hace imposible el diagnóstico clínico sin el concurso del laboratorio. Pero este comportamiento «silente» contrasta con la gran cantidad de complicaciones a las que puede dar lugar y la importancia de las mismas. Así, representa la primera causa de enfermedad inflamatoria pélvica y por ello de esterilidad de origen tubárico, estimándose que hasta un $75 \%$ de las infertilidades de este tipo tendrían su origen en una infección desapercibida en el pasado por $\mathrm{CT}^{1.4}$.

Desde el punto de vista de morbimortalidad materno-fetal, la infección redunda en una mayor incidencia de embarazo extrauterino, abortos de repetición, rotura prematura de membranas, parto pretérmino, endometritis postparto y conjuntivitis y neumonía en el recién nacido ${ }^{1.4}$.

Aunando ambos puntos de vista - clínico y epidemiológico - se constata la gran importancia de la infección por CT que se traduce en la gran carga económica que supone para la sociedad $^{3}$.

Por ello, la mayoría de autores sugieren la conveniencia de realizar programas de prevención secundaria mediante cribados ${ }^{3.5-8}$, ya que concurren los principales criterios para realizarlos $^{9-11}$ y así mismo, disponer actualmente de técnicas diagnósticas asequibles y ampliamente difundidas, que permiten el procesado de gran cantidad de muestras en poco tiempo y a relativo bajo $\operatorname{coste}^{12}$. El principal objetivo de este estudio es llevar a cabo una primera fase en la evaluación del programa de cribado de infección asintomática por CT en las mujeres que acuden a un centro de planificación familiar.

\section{MATERIAL Y MÉTODOS}

En el año 1990 se puso en funcionamiento un programa de detección de infección asintomática por CT entre las mujeres que acuden al Centro de Planificación Familiar Miguel Servet de La Coruña, gracias a la colaboración entre este centro y el Servicio de Medicina Preventiva del Hospital Juan Canalejo de dicha localidad.

Un estudio epidemiológico basal había cifrado la prevalencia global de dicha infección en $5.1 \%$,entre las mujeres que acudieron a dicho centro en el año $1990^{13}$.

El objetivo general del programa era conseguir una disminuición en dicha prevalencia. A nivel específico se plantcaron como objetivos operativos lograr un descenso de la prevalencia en el primer año (1990-1991) de alrededor del $50 \%$, y ésta disminuirla a la mitad en los dos años siguientes (1991-1993), con lo que se pretendía alcanzar al final del período una prevalencia de entre $1.2 \%$ y $1.3 \%$.

Pacientes: En el estudio se incluyeron un total de 6.746 mujeres, mayores de 15 años, sexualmente activas, provenientes del área sanitaria de La Coruña, que acudieron durante el período de 1990 a 1993 al Centro de Planificación Familiar Miguel Servet de La Coruña para consejo de anticoncepción y/o examen ordinario.

Método: Entrevista y exploracion por dos ginecólogos con experiencia en este campo, se realizó una ficha epidemiológica recogiendo toda la información referente a las variables de interés (edad, estado civil, profesión, método anticonceptivo y residencia). Se tomó una muestra del canal endocervical que se remitía al servicio de Medicina Preventiva del Hospital Juan Canalejo donde se procesaban para la detección de CT mediante la técnica diagnóstica Sonda DNAProbe $^{12}$. Los resultados positivos se refieren al Centro de Planificación donde se prescribió tratamiento antibiótico adecuado a la enferma y a $\mathrm{su} / \mathrm{s}$ pareja/s sexuales, se facilitó información sobre las E.T.S. y pautas de comportamiento sexual saludables. Al cabo de a un mes se realizó un nuevo examen para verificar su estado.

Para realizar la primera fase de la evaluación del programa se procedió en primer lugar a la introducción en una base de datos informática de la información correspondiente al período 91-93 y a unirla a la del año 90 ya disponible en este so- 
porte. Dado que se pretendía evaluar la evolución de la prevalencia a lo largo del tiempo de estudio, se realizó un estudio transversal para cada año, tanto a nivel global como para cada uno de los estratos en que se dividió a población estudiada en función de las variables edad, estado civil, método anticonceptivo, residencia y profesión (las mismas que se utilizaron en el estudio previo del año 90, para lograr una máxima comparabilidad). Posteriormente se analizó la existencia y magnitud de la evolución seguida por la prevalencia en esos tres años y en la misma línea se valoró la efectividad del programa cuantificada en términos de la reducción proporcional de infección, determinada por la expresión : (P90P93)/P90, donde P90 representa la prevalencia en el año 90, y P93 la del año 93 $3^{10,14}$.

Análisis estadístico: En el estudio descriptivo de cada uno de los años se aplicó la prueba de la Ji-cuadrado con la corrección de Yates, y la prueba de Cornfield o el método exacto cuando fue necesario para la construcción de los intervalos de confianza. Para el análisis de la evolución temporal de las prevalencias se utilizó la prueba de la Chi-cuadrado para tendencias de proporciones (extensión de Mantel) ${ }^{15,16}$.

También se realizó un análisis multivariante mediante regresión logística (método forward) para el primer y último año y se valoró la tendencia de la prevalencia en el global de la población en el período estudiado por el mismo método, incluyendo para ello la variable «año de estudio» ${ }^{17}$.

Para la confección de la base de datos se utilizó el paquete informático Epi Info versión $5.0^{16}$. Los cálculos estadísticos fueron realizados mediante los programas SPSS ${ }^{17}$ y Epi Info v.5.0.

\section{RESULTADOS}

Las características de la población apenas sufren variaciones durante el período estudiado. Destaca únicamente el gran salto en la proporción de mujeres que declaran utilizar métodos anticonceptivos de barrera entre el año $90(2.2 \%)$ y el 91 $(18.7 \%)$, manteniéndose en un nivel similar al del 91 en los dos años restantes (Tabla 1).

Tabla 1

Características de la población estudiada años 1990, 1991, 1992, 1993

\begin{tabular}{|c|c|c|c|c|c|c|c|c|}
\hline \multirow[b]{2}{*}{ EDAD } & \multicolumn{2}{|c|}{1990} & \multicolumn{2}{|c|}{1991} & \multicolumn{2}{|c|}{1992} & \multicolumn{2}{|c|}{1993} \\
\hline & $\mathbf{N}$ & $\%$ & $\mathbf{N}$ & $\%$ & $\mathbf{N}$ & $\%$ & $\mathbf{N}$ & $\%$ \\
\hline $15-21$ & 361 & 15.0 & 245 & 11.9 & 258 & 11.8 & 311 & 12.5 \\
\hline $22-28$ & 711 & 29.6 & 636 & 30.9 & 621 & 28.3 & 707 & 28.4 \\
\hline $29-35$ & 684 & 28.5 & 588 & 28.6 & 581 & 26.5 & 681 & 27.3 \\
\hline$>35$ & 642 & 26.7 & 589 & 28.6 & 731 & 33.4 & 791 & 31.8 \\
\hline \multicolumn{9}{|l|}{ ESTADO CIVIL } \\
\hline Soltera & 590 & 25.3 & 493 & 24.2 & 592 & 27.3 & 704 & 28.8 \\
\hline Casada & 1620 & 69.5 & 1414 & 69.4 & 1445 & 66.5 & 1558 & 63.7 \\
\hline Otros & 121 & 5.2 & 130 & 6.4 & 135 & 6.2 & 183 & 7.5 \\
\hline \multicolumn{9}{|l|}{ ANTICONCEPCION } \\
\hline Orales & 642 & 27.0 & 578 & 28.5 & 673 & 31.0 & 752 & 31.3 \\
\hline DIU & 1007 & 42.4 & 885 & 43.6 & 956 & 44.5 & 1000 & 41.6 \\
\hline Barrera & 54 & 2.2 & 380 & 18.7 & 394 & 18.4 & 484 & 20.1 \\
\hline Otros & 671 & 28.3 & 185 & 9.1 & 124 & 5.8 & 167 & 6.9 \\
\hline \multicolumn{9}{|l|}{ RESIDENCIA } \\
\hline Urbana & 1717 & 75.8 & 1475 & 72.0 & 1547 & 71.2 & 1694 & 68.7 \\
\hline Rural & 547 & 24.2 & 574 & 28.0 & 626 & 28.8 & 773 & 31.3 \\
\hline \multicolumn{9}{|l|}{ PROFESION } \\
\hline Titulada superior & 58 & 2.59 & 49 & 2.4 & 73 & 3.4 & 77 & 3.2 \\
\hline Titulada media & 123 & 5.5 & 110 & 5.4 & 102 & 4.7 & 100 & 4.2 \\
\hline Obrera cualificada & 539 & 24.1 & 472 & 23.2 & 412 & 19.0 & 461 & 19.3 \\
\hline Obrera no cualificada & 301 & 13.4 & 455 & 22.4 & 584 & 27.0 & 691 & 29.0 \\
\hline Ama de casa & 911 & 40.7 & 745 & 36.7 & 741 & 34.3 & 741 & 31.1 \\
\hline Estudiante & 304 & 13.5 & 201 & 9.9 & 251 & 11.6 & 315 & 13.2 \\
\hline TOTAL & 2408 & & 2060 & & 2192 & & 2494 & \\
\hline
\end{tabular}


Los resultados del estudio previo a la puesta en marcha del programa de cribado mostraban una prevalencia global de infección asintomática por C. trachomatis de $5.1 \%$ entre las mujeres estudiadas. Sólo el pertenecer al grupo de las más jóvenes (15 a 21 años): $O R=1.67$ $(1.04,2.65)$ y al grupo de obreras no cualificadas en cuanto a la profesión: $O R=1.66$ $(1.00,2.72)$, comportaba un riesgo estadísticamente significativo ${ }^{13}$.

En cuanto a la evolución de la prevalencia global a lo largo de los tres años estudiados, se constata un descenso significativo, alcanzándose una cifra de $2.8 \%$ en el año $91,2.0 \%$ en el año 92 y $1.0 \%$ en el 93 ( $p<0.00)$ (Tabla 2).

Los resultados de la tendencia de la prevalencia de C.trachomatis ajustada por cada variable muestran una tendencia descendente prácticamente idéntica entre sí y al global a lo largo del período estudiado (Tabla 3 ).

Cuando se evalúa la tendencia de la prevalencia considerada por los distintos estratos, se constata un descenso significativo a todos los niveles a excepción del grupo que utilizan métodos anticonceptivos de barrera y las tituladas superiores (Tabla 4).
Todos estos datos son asumibles cuando se utiliza el análisis multivariante para estudiar la tendencia temporal en el período estudiado, introduciendo la variable «año», además de las otras, ya que se obtiene un modelo de regresión donde la única variable incluída es ésta.

En el análisis comparativo entre el último de la realización del programa (1993) y el de referencia (1990) destaca la disminución de la prevalencia global a una quinta parte y se verifica una asociación estadística entre la inclusión en el cribado y una baja probabilidad de presentar una infección por C. trachomatis, tanto a nivel global, $O R=0.24(0.15-0.38)$, como realizando la comparación por las distintas variables, a excepción del grupo que utilizaba métodos anticonceptivos de barrera, y las tituladas superiores y medias en cuanto a profesión (Tabla 5).

La efectividad del programa se cifra a nivel global en un $80 \%$, destacando de entre las mujeres que presentaban un descenso significativo de la prevalencia, las obreras no cualificadas y las estudiantes $(89.5 \%$ y $89.2 \%$, respectivamente) (Tabla 6).

Tabla 2

Análisis de la tendencia temporal de prevalencia de chlamydia trachomatis 1990-1993

\begin{tabular}{|cccccc|}
\hline & Positivo & Negativo & Total & Prevalencia (\%) & O.R. \\
\hline 1990 & 123 & 2285 & 2408 & 5,11 & 1,00 \\
1991 & 58 & 2002 & 2060 & 2,82 & 0,54 \\
1992 & 43 & 2149 & 2192 & 1,96 & 0,37 \\
1993 & 25 & 2469 & 2494 & 1,00 & 0,19 \\
\hline
\end{tabular}

$\mathbf{P}=0,00$

2 para tendencias de proporciones (extensión de Mantel) $=80.683$

Tabla 3

Odds Ratios ajustados por cada una de las variables

\begin{tabular}{|cccccc|}
\hline & Edad & Estado civil & Anticoncepción & Residencia & Profesión \\
\hline 1990 & $1,00^{*}$ & $1,00^{*}$ & $1,00^{*}$ & $1,00^{*}$ & $1,00^{*}$ \\
1991 & 0,55 & 0,53 & 0,61 & 0,54 & 0,52 \\
1992 & 0,39 & 0,36 & 0,38 & 0,36 & 0,34 \\
1993 & 0,20 & 0,18 & 0,22 & 0,19 & 0,20 \\
& P: 0,00 & P: 0,00 & P: 0,00 & P: 0,00 & $P: 0,00$ \\
\hline
\end{tabular}

2 para tendencias de proporciones (extensión de Mantel) $=80.683$ 
Tabla 4

Análisis de la tendencia temporal de prevalencia de chlamydia trachomatis 1990-1993 en función de cada una de las variables

\begin{tabular}{|c|c|c|c|c|c|c|c|c|c|}
\hline & & & & & & & & & \\
\hline EDAD & $\underset{\mathrm{V}}{\mathrm{PRE}}$ & O.R. & $\begin{array}{c}\text { PRE } \\
\text { V }\end{array}$ & O.R. & $\begin{array}{c}\text { PRE } \\
\text { V }\end{array}$ & O.R. & $\begin{array}{c}\text { PRE } \\
V\end{array}$ & O.R. & $\mathrm{P}$ \\
\hline $15-21$ & 7.47 & 1.00 & 3.26 & 0.42 & 2.33 & 0.29 & 1.29 & 0.16 & 0.00 \\
\hline 22.28 & 4.64 & 1.00 & 2.99 & 0.63 & 2.47 & 0.58 & 1.27 & 0.26 & 0.00 \\
\hline $29-35$ & 4.82 & 1.00 & 2.38 & 0.48 & 1.72 & 0.35 & 1.03 & 0.20 & 0.00 \\
\hline$>35$ & 4.36 & 1.00 & 2.89 & 0.65 & 1.37 & 0.30 & 0.63 & 0.14 & 0.00 \\
\hline ESTADO CI & & & & & & & & & \\
\hline Soltera & 6.60 & 1.00 & 3.04 & 0.44 & 3.38 & 0.49 & 1.42 & 0.20 & 0.00 \\
\hline Casada & 4.40 & 1.00 & 2.69 & 0.59 & 1.38 & 0.30 & 0.77 & 0.17 & 0.00 \\
\hline Otros & 8.30 & 1.00 & 3.85 & 0.44 & 2.22 & 0.25 & 1.64 & 0.18 & 0.00 \\
\hline ANTICONC & ION & & & & & & & & \\
\hline Orales & 4.20 & 1.00 & 2.77 & 0.49 & 2.53 & 0.41 & 1.59 & 0.26 & 0.00 \\
\hline DIU & 4.10 & 1.00 & 3.16 & 0.77 & 1.78 & 0.43 & 0.60 & 0.14 & 0.00 \\
\hline Barrera & 1.85 & 1.00 & 1.84 & 0.99 & 2.28 & 1.24 & 0.62 & 0.33 & 0.00 \\
\hline Otros & 5.96 & 1.00 & 3.24 & 0.53 & 0.00 & 0.00 & 1.80 & 0.29 & 0.00 \\
\hline RESIDENCI & & & & & & & & & \\
\hline Urbana & 5.06 & 1.00 & 3.46 & 0.67 & 1.81 & 0.35 & 1.12 & 0.21 & 0.00 \\
\hline Rural & 5.30 & 1.00 & 1.04 & 0.19 & 2.24 & 0.41 & 0.65 & 0.12 & 0.00 \\
\hline PROFESION & & & & & & & & & \\
\hline Tit. superior & 3.57 & 1.00 & 0.00 & 0.00 & 1.37 & 0.39 & 0.00 & 0.00 & 0.12 \\
\hline Tit. media & 4.23 & 1.00 & 0.91 & 0.22 & 1.96 & 0.47 & 0.00 & 0.00 & 0.04 \\
\hline Obrera cual. & 5.68 & 1.00 & 2.54 & 0.46 & 2.67 & 0.48 & 1.95 & 0.35 & 0.00 \\
\hline Obr. no cual. & 8.27 & 1.00 & 2.42 & 0.30 & 2.40 & 0.30 & 0.87 & 0.11 & 0.00 \\
\hline Ama de casa & 4.71 & 1.00 & 3.49 & 0.77 & 0.94 & 0.20 & 1.08 & 0.23 & 0.00 \\
\hline Estudiante & 5.91 & 1.00 & 2.98 & 0.59 & 2.39 & 0.47 & 0.64 & 0.12 & 0.00 \\
\hline
\end{tabular}

Tabla 5

Comparación global y por variables de la prevalencia. Años 1993-1990

\begin{tabular}{|c|c|c|c|c|}
\hline & Prevalencia & $O . R$. & IC. $95 \%$ & $\begin{array}{c}1990 \\
\text { Prevalencia }\end{array}$ \\
\hline \multicolumn{5}{|l|}{ EDAD } \\
\hline $15-21$ & 1.29 & 0.16 & $0.40-0.47$ & $7.47^{\circ}$ \\
\hline $22-28$ & 1.27 & 0.26 & $0.12-0.58$ & 4.64 \\
\hline $29-35$ & 1.03 & 0.20 & $0.08-0.48$ & 4.84 \\
\hline$>35$ & 0.63 & 0.14 & $0.04-0.37$ & 4.36 \\
\hline \multicolumn{5}{|l|}{ ESTADO CIVIL } \\
\hline Soltera & 1.42 & 0.20 & $0.09-0.43$ & 6.60 \\
\hline Casada & 0.77 & 0.17 & $0.09-0.32$ & 4.40 \\
\hline Otros & 1.64 & 0.18 & $0.03-0.74$ & 8.30 \\
\hline \multicolumn{5}{|l|}{ ANTICONCEPCION } \\
\hline Orales & 1.59 & 0.26 & $0.13-0.52$ & 4.20 \\
\hline DIU & 0.60 & 0.14 & $0.05-0.34$ & 4.10 \\
\hline Barrera & 0.62 & 0.33 & $0.03-17.68$ & 1.85 \\
\hline Otros & 1.80 & 0.29 & $0.06-0.93$ & 5.96 \\
\hline \multicolumn{5}{|l|}{ RESIDENCIA } \\
\hline Urbana & 1.12 & 0.21 & $0.12-0.36$ & 5.06 \\
\hline Rural & 0.65 & 0.12 & $0.04-0.31$ & 5.30 \\
\hline \multicolumn{5}{|l|}{ PROFESION } \\
\hline Titulada superior & 0.00 & 0.00 & $0.00-3.99$ & 3.57 \\
\hline Titulada media & 0.00 & 0.00 & $0.00-1.32$ & 4.23 \\
\hline Obrera cualificada & 1.95 & 0.35 & $0.15-0.78$ & 5.68 \\
\hline Obrera no cualificada & 0.87 & 0.11 & $0.04-0.27$ & 8.57 \\
\hline Ama de casa & 1.08 & 0.23 & $0.10-0.52$ & 4.71 \\
\hline Estudiante & 0.64 & 0.12 & $0.01-0.54$ & 5.91 \\
\hline GLOBAL & 1.00 & 0.24 & $0.15-0.38$ & 5.10 \\
\hline
\end{tabular}


Tabla 6

Efectividad del programa

\begin{tabular}{|lc|}
\hline \multicolumn{1}{|c|}{ Prevalencia } & Efectividad \\
\hline EDAD & \\
$15-21$ & 82,8 \\
$22-28$ & 72,6 \\
$29-35$ & 78,6 \\
$>35$ & 85,5 \\
ANTICONCEPCION & \\
Orales & 62,1 \\
DIU & 85,3 \\
Otros & 69,8 \\
RESIDENCIA & \\
Urbana & 77,9 \\
Rural & 87,7 \\
ESTADO CIVIL & \\
Soltera & \\
Casada & 67,8 \\
Otros & 77,5 \\
PROFESION & 80,2 \\
Obrera cualificada & \\
Obrera no cualificada & 65,6 \\
Ama de casa & 89,8 \\
Estudiante & 77,1 \\
GLOBAL & 89,1 \\
\hline
\end{tabular}

\section{DISCUSIÓN}

Transcurridos tres años desde el comienzo del programa de cribado la prevalencia global de infección por $\mathrm{C}$. Trachomatis ha descendido en aproximadamente un $80 \%$ (de $5.1 \%$ en 1990 a $1.0 \%$ en 1993), en la línea de lo referido en estudios similares en Estados Unidos ${ }^{18-20}$, Suecia ${ }^{5}$ y Francia $^{21}$.

El análisis de este descenso ajustado por cada una de las variables denota una tendencia decreciente a lo largo del período estudiado, prácticamente idéntica a la observada en la valoración global, lo que demuestra su relación con el programa. Esto viene avalado por el análisis multivariante donde sólo la variable «año de estudio» se relaciona con la prevalencia de infección, al ser la única significativa para entrar en el modelo de regresión logística.

Igualmente se mantuvieron durante el programa las mismas características en cuanto a personal y técnicas en la recogida de la información y de las muestras, y su procesamiento, por lo que tampoco pueden haber afectado en modo alguno a los resultados.
Se observa un descenso significativo de la prevalencia en todos los grupos, a excepción de las tituladas superiores, debido a las pocas mujeres incluídas, y las mujeres que usan métodos de barrera como técnica contraceptiva, ya que supone de por sí, una protección frente a la infección, de manera que el impacto del programa en este grupo es bajo. En la población estudiada el uso de métodos de barrera es perfectamente identificable con la utilización de preservativo, ya que de las 1.312 mujeres incluidas en esta categoría, $1.303(99.3 \%)$ refirieron tal método y el resto, otros (diafragma y esponja). Sin embargo, la inclusión en este grupo no garantiza el uso sistemático del preservativo, ya que abarca un espectro que va desde las mujeres que lo utilizan en todas sus relaciones sexuales hasta las que lo hacen esporádicamente (sólo días fértiles, sólo al final del coito), lo que debe ser tenido en cuenta al considerarlo como práctica protectora de contagio de E.T.S. El gran salto existente en las categorías «preservativo» $y$ «otros» entre el año 1990 y 1991 no puede ser sólo explicado por la faceta informativa del programa acerca del uso del condón como método efectivo de prevención de E.T.S., o por las campañas institucionales para la prevención del Sida, sino que fue debido en su mayor parte a un cambio en el criterio de inclusión en uno u otro grupo.

Las mujeres con las mismas características en cuanto a riesgo para CT en el año 90 y 93, eran menos vulnerables a ser infectadas, así las dos categorías identificadas en el año 90 con un riesgo significativo de padecer una infección por CT (edad de 15 a 21 años y obreras no cualificadas) pasan a presentar riesgos no significativos en el año 93. Esto sugiere que el programa es en gran modo responsable de la disminución de la prevalencia observada.

El diseño del estudio, donde la propia población antes del programa funciona como control de sí misma, hace que sea difícil deslindar que proporción de la disminución de la prevalencia es achacable al programa o a otros factores que pudieran estar actuando, ${ }^{10,22}$ pero en la práctica surgen muchas limitaciones de orden técnico, económico y ético que impiden disponer de un grupo control. De hecho, la práctica totalidad de estudios revisados utilizan un diseño similar. Además no existe ninguna referencia a disminuciones sin llevar a cabo intervenciones como ésta y mucho menos de la magnitud observada. 
Teniendo esto en cuenta, la efectividad del programa ${ }^{10.14}$ de cribado puede ser cuantificada en términos de la reducción proporcional de la infección que en nuestro caso es de un $82 \%$ a nivel global. El estudio parte de una distribución bastante homogénea de la prevalencia en los diferentes grupos, a excepción de las cifras más elevadas en el grupo de edad de 15 a 21 años $(7.47 \%)$, la categoría «otros» de estado civil $(8.3 \%)$ y obreras no cualificadas $(8.3 \%)$, y por abajo las incluidas en la categoria «barrera» $(1.85 \%)$ y las tituladas superiores $(3.5 \%)$, hallándose el resto en un rango comprendido entre $4.1 \%$ y $6.6 \%$. Los datos del año 93 dan una distribución todavía más homogénea de la prevalencia en los grupos estudiados, estando todos los valores obtenidos en un intervalo desde $0.6 \%$ a $1.8 \%$, en los que mostraron un descenso significativo de la prevalencia entre el primer y último año.

Ello es debido a la diferente forma en que inciden en cada grupo los factores que condicionan la efectividad ${ }^{10}$, de modo que va desde un máximo en la categoría de las obreras no cualificadas $(89.5 \%)$ hasta un mínimo en las del grupo de anticoncepción oral $(62.1 \%)$ : Por un lado, influyen por igual en todos los grupos la sensibilidad y especificidad del método diagnóstico ${ }^{11,12}$, que se mantienen constantes al utilizarse siempre el mismo test y ser características inherentes a éste. Igualmente ocurre con el acuerdo entre el diagnóstico y el tratamiento (probabilidad de que se paute un correcto tratamiento asumiendo un correcto diagnóstico), ya que la pauta de tratamiento está protocolizada y es seguida por el personal facultativo del centro en la totalidad de los casos. Por otro lado, entre los factores que tienen diferente influencia está el valor predictivo positivo del test", que depende directamente de la prevalencia, por lo que varía entre los grupos, y análogamente va a ir disminuyendo conforme la actuación va teniendo éxito y hace disminuir la prevalencia, de modo que se incrementa la importancia relativa de los falsos positivos. Otro elemento es el grado de cumplimiento del paciente de las medidas terapéuticas y preventivas dadas por el médico, que si bien se estima como de alto grado, no lo es por igual en todos los grupos. También influye el nivel de cobertura del tratamiento o de las medidas preventivas en la población de interés, ya que si bien el programa contempla en su diseño el tratamiento de la mujer infectada y de su/s pareja/s sexuales, su cumplimiento es muy variable. A este respecto se podría echar en falta que no se recabe información sobre el número de parejas sexuales de cada mujer, pero las características sociológicas de la población estudiada y de la técnica de recogida de los datos, que no permite el anonimato, aconsejaron la no inclusión de esta variable, ya que la fiabilidad de las respuestas sería muy baja y supondrían un sesgo muy importante en esta cuestión.

Como conclusiones finales cabe destacar que el programa demuestra una efectividad más que aceptable en prácticamente todos los grupos estudiados. Obviamente, incluir a toda la población constituye la mejor alternativa en cuanto a detectar el máximo de casos, pero también la más cara, y hace que se parta de la prevalencia más baja posible, lo cual va en detrimento del valor predictivo positivo y por ende de su eficiencia, ya que conforme avanza el programa el coste por caso también aumenta. Pero en el diseño del programa influyeron ciertas condiciones locales junto con los criterios puramente económicos. Así, la información registrada no permite estratificar en función de múltiples variables y puede suponer una perdida de capacidad para identificar grupos de riesgo, pero hace mucho más ágil y adaptado a las posibilidades la realización del cribado. También se consideró que la prevalencia de partida era alta en el contexto del país, y las circunstancias, antes mencionadas, que concurren en el grupo «método de barrera», que llevó a incluirlas en el programa aún presentando una prevalencia notablemente más baja. Finalmente, también infiuyó en el diseño la disponibilidad existentente de recursos técnicos, económicos y humanos. No obstante, ante la posibilidad de implementar programas similares en otros centros del área, sería adecuado completar la evaluación con un análisis que confronte los resultados y costes del programa con el ahorro que conlleva disminuir la prevalencia de esta infección para cuantificar su eficiencia, a nivel global y en los diferentes grupos, en función de la efectividad mostrada. Ello conformaría la base para realizar, si fuese necesario, modificaciones encaminadas a mejorar el rendimiento del programa.

\section{BIBLIOGRAFÍA}

1. Hunter Handsfield $H$. Avances recientes en las enfermedades de transmisión sexual (I). Enfermedades bacterianas. Hosp pract 1992; 7 (2): 39-49. 
2. Center for Diseases Control. Progress toward achieving the national 1990 objectives for sexually trasmitted diseases. JAMA 1987; 257 : 2141-2143.

3 Center for Diseases Control. Recommendations for the prevention and management of chlamydia trachomatis infections. MMWR 1993; 42, RR12:1-39.

4. García de Salazar Fernández JC, Elola Oyarzabal B. Enfermedades venéreas producidas por el género chlamydia. Gac Med Bilbao 1988; 85(3-4); 109-117.

5. Ripa T. Epidemiologyc control of genital chlamydia trachomatis infections. Scand J Infect Dis 1990; 69: 157-167.

6. Weinstock HS, Bolan GA, Balladares C, Back A, Oliva $\mathrm{G}$. Chlamydia trachomatis infection in women: a need for universal screening in high prevalence populations?. Am J Epidemiol 1992; 135(1): 41-47.

7. Phillips RS, Aronson MD, Taylor WC, Safran C. Should tests for chlamydia trachomalis cervical infection be done during routine gynecologic visits?. Ann Intern Med 1987; 107: 188-194.

8. Stergachis A, Scholes D, Heidrich FE, Sherer DM, Holmes KK, Stamm W. Selective screening for chlamydia trachomatis infection in a primary care population of women. Am J Epidemiol 1993; $138(3): 143-153$.

9. Hunter Hansfield H; Jasman LL; Roberts PL, Hanson VW, Kothenbeutel RL, Stamm WE. Criteria for selective screening for chlamydia trachomatis infection in women attending family planning clinics. JAMA 1986; 225(13): 17301734.

10. Pineault R, Daveluy C. La planificación sanitaria. conceptos, métodos, estrategias. Barcelona: Masson, 1989.

11. González Enríquez J, Banegas Banegas JR, Martín Moreno J, Rodriguez Artalejo F, Villar Alvarez F. Criterios para la realización de programas de detección precoz de enfermedad en la población. Med Integr 1989; 13(4): 199-204.
12. Rosales $\mathrm{M}$, Domínguez V, Bonacho $\mathrm{Y}$, Vidal X. Diagnóstico rápido de chlamydia trachomatis por sonda ADN-Probe en una población con bajo riesgo de infección. Fnferm Infecc Microbiol Clin 1992; 10(2): 111-114.

13. Rosales M, Domínguez V, Bonacho Y, Vidal X. Epidemiología de chlamydia trachomatis en una población que acude a un centro de planificación familiar. Rev Clin Esp 1993; 192(9): 424-427.

14. Sellors JW, Pickard L, Gafni A, Goldsmith $\mathrm{CH}$, Mahony JB, Chernesky MA. Effecttiveness and efficiency of selective vs universal screening for chlamydial infection in sexually active young women. Arch Intern Med 1992; 152: 1837-1844.

15. Schlesselman JJ. Case-Control studies. New York: Oxford University Press, 1982.

16. Dean AG, Dean JA, Burton AH, Dicker RC. Epi Info versión 5 a word proccesor and database and statistic program for epidemiology on microcomputer. Stone mountain, Georgia: USD Inc, 1990.

17. Alvarez Cáceres R. Estadística multivariante y no paramétrica con SPSS. Aplicación a las ciencias de la salud. Madrid: Díaz de Santos, 1994.

18. Addis DG, Vaughn ML, Ludka D, Pfister J, Davis JP. Decreased prevalence of chlamydia infection associated with a selective screening program in family planning clinics in Wisconsin. Sex Transm Dis 1993: 28-35.

19. Chlamydia prevalence and screening practices. San Diego county, California. MMWR 1993; 43: 366-375.

20. Willard MA, Edman RL. Screening for genital chlamydia trachomatis in female patients in primary care setting. J Fam Pract 1989; 28(1): 9798.

21. Job Spira N, Meyer L, Bouvet E; Janaud A, Spira A. The prevention of sexually trasmitted diseases which affect fertility: methodological problems and initial results. Eur J Obstet Gynecol 1988; 27 : $157-164$

22. Donabedian A. La calidad de la atención médica. Definición y métodos de evaluación. México: La prensa médica mexicana S.A., 1984. 